\section{What's up with Golden Retrievers under primary veterinary care in the UK}

\section{Jean Lester, Dan G O'Neill, David B Church, Dave C Brodbelt}

Royal Veterinary College, London, United Kingdom

\section{OBJECTIVES}

Golden Retrievers (GRs) are a popular UK breed that are consistently in the top 10 Kennel Club registrations. This study aimed to describe demography and disorder prevalence of GRs under UK primary veterinary care.

\section{METHODS}

GRs under primary veterinary care in the VetCompass database during 2013 were identified. Demography was described. Clinical records of a random sample were reviewed to manually extract disorder and mortality information.

\section{RESULTS}

The study included 5439 GRs (1.2\% of all dogs). The median age was 6.2 years (IQR 2.8-9.7). The median adult bodyweight for females was $34.2 \mathrm{~kg}$ (IQR 30.638.0) and males 38.1kg (IQR 34.2-42.5). Disorder data was extracted on 3999 GRs. The most common specific disorder terms were overweight/obesity $(9.9 \%$, 95\% Cl 9.0-10.9), otitis externa (8.1\% 95\% Cl 7.2-8.9), osteoarthritis $(7.5 \%, 95 \% \mathrm{Cl} 6.7-8.3)$, periodontal disease $(7.2 \%, 95 \% \mathrm{Cl} 6.4-8.0)$, diarrhoea $(4.9 \%, 95 \% \mathrm{Cl}$ $4.3-5.6)$ and anal sac impaction $(3.8 \%, 95 \% \mathrm{Cl} 3.3-$ 4.5). There were 589 deaths during the study. Longevity of males (12.4 years, IQR 10.2-13.5) was similar to females (12.4 years, IQR 10.5-14.0). The most common disorder groups as a cause of death were spinal disorders (15.2\%, 95\% Cl 12.1-18.6), neoplasia (13.2\%, $95 \% \mathrm{Cl} 10.3-16.5)$, mass-associated disorders $(9.6 \%$, $95 \% \mathrm{Cl} 7.1-12.5)$ and brain disorders $(8.8 \%, 95 \% \mathrm{Cl}$ 6.5-11.6).

\section{STATEMENT (CONCLUSIONS)}

Breed selection is reported to lead to inbreeding with loss of genetic diversity, increased expression of inherited defects and breed predisposition to disorders. These results provide generalisable insights to the health of UK GRs that can support breed health policy and welfare reform. 